\title{
A novel process for the production of Triethylene glycol di-2-ethylhexoate by reactive distillation using a sulfated zirconia catalyst
}

\author{
Xingang Li, Qingyu Zhang, Hong Li, Xin Gao*
}

School of Chemical Engineering and Technology, Tianjin University, National

Engineering Research Center of Distillation Technology, Collaborative Innovation

Center of Chemical Science and Engineering(Tianjin), Tianjin 300072, China

* Corresponding author E-mail: gaoxin@tju.edu.cn 
Table S1. UNIQUAC model parameters

\begin{tabular}{llllll}
\hline Component $\mathrm{i}$ & Water & Water & Water & Water & TEG \\
Component $\mathrm{j}$ & 2-ETH & TEG & TGDE & TGME & 2-ETH \\
Source & APV100 LLE-LIT & NISTV100NIS T-HOC & R-PCES & R-PCES & R-PCES \\
$\mathrm{A}_{\mathrm{ij}}$ & 0 & 0.3925 & 0 & 0 & 0 \\
$\mathrm{~A}_{\mathrm{ji}}$ & 0 & -0.05207 & 0 & 0 & 0 \\
$\mathrm{~B}_{\mathrm{ij}}$ & -270.83 & 237.479 & -320.512 & -886.371 & 94.19 \\
$\mathrm{~B}_{\mathrm{ji}}$ & -426.03 & -1008.78 & -26.3835 & 345.132 & -142.706 \\
Component $\mathrm{i}$ & TEG & TEG & 2 -ETH & 2 -ETH & TGDE \\
Component j & TGDE & TGME & TGDE & TGME & TGME \\
Source & R-PCES & R-PCES & R-PCES & R-PCES & R-PCES \\
$\mathrm{A}_{\mathrm{ij}}$ & 0 & 0 & 0 & 0 & 0 \\
Aji & 0 & 0 & 0 & 0 & 0 \\
Bij & 66.9757 & 54.3989 & 159.206 & 82.5105 & -65.9968 \\
Bji & -240.371 & -122.045 & -212.349 & -65.3413 & 45.3933 \\
\hline
\end{tabular}

APV100 LLE-LIT, NISTV100NIS T-HOC, built-in parameter provided by Aspen Plus data base. R-PCES, Missing parameters estimated by UNIFAC method.

Table S2. Summary of column parameters.

\begin{tabular}{ll}
\hline Variable name & Value \\
\hline Pressure (atm) & 1 \\
Theoretical stages of rectifying section & 3 \\
Theoretical stages of reaction section & 30 \\
Theoretical stages of stripping section & 2 \\
Residence time per stages in reaction section (min) & 5 \\
Feeding stage of TEG and 2-ETH & 4,4 \\
Mass reflux ratio & 1.1 \\
Feed rate $(\mathrm{kg} / \mathrm{h})$ & 1315.8 \\
Mole ratio of 2-ETH and TEG in the feed & $2: 1$ \\
Distillate rate $(\mathrm{kg} / \mathrm{h})$ & 112 \\
Reboiler duty $(\mathrm{kW})$ & 441.985 \\
\hline
\end{tabular}




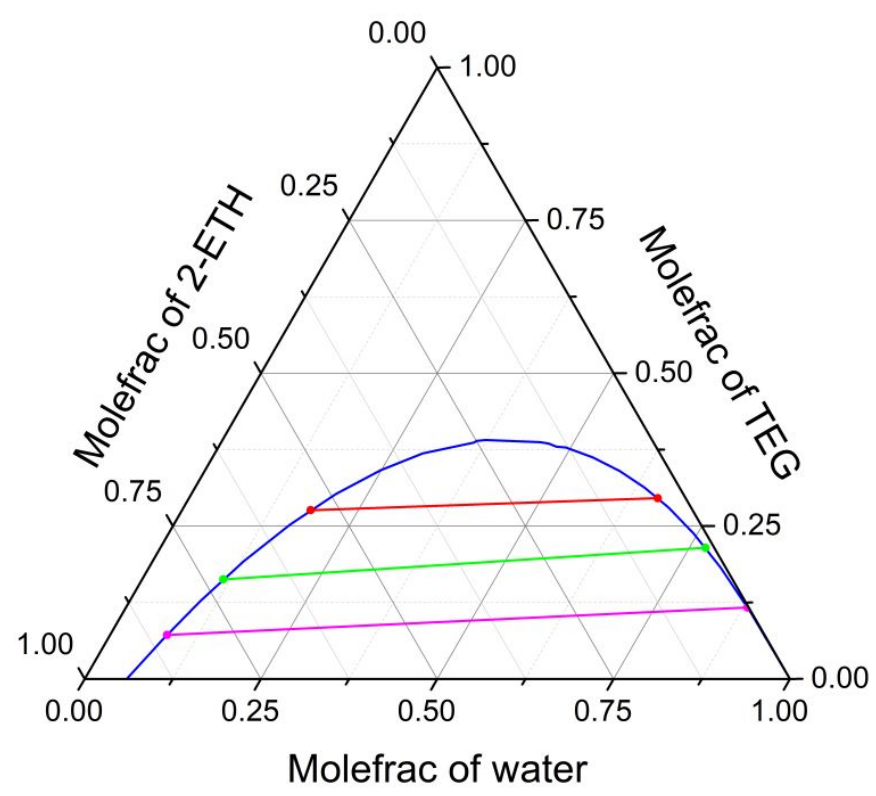

Figure S1. Ternary diagram for Water/TEG/2-ETH at $298.15 \mathrm{~K}$ and atmospheric pressure. ${ }^{\text {a }}$

a The data in the Aspen Plus v8.8. 